Article

\title{
FMTPen: A Miniaturized Handheld Fluorescence Molecular Tomography Probe for Image-Guided Cancer Surgery
}

\author{
Hao Yang ${ }^{1}$, Bin He ${ }^{1}$, Xianjin Dai ${ }^{1}$, Minati Satpathy ${ }^{2}$, Lily Yang ${ }^{2}$ and Huabei Jiang ${ }^{1, *}$ \\ 1 Department of Biomedical Engineering, University of Florida, Gainesville, FL 32611, USA; \\ E-Mails: hao.yang@ufl.edu (H.Y.); hebin03@gmail.com (B.H.); xjdai@ufl.edu (X.D.) \\ 2 Department of Surgery, Emory University, Atlanta, GA 30322, USA; \\ E-Mails: msatpat@emory.edu (M.S.); lyang02@emory.edu (L.Y.) \\ * Author to whom correspondence should be addressed; E-Mail: hjiang@bme.ufl.edu; \\ Tel.: +1-352-273-9336; Fax: +1-352-273-9221.
}

Received: 19 February 2015 / Accepted: 10 March 2015 / Published: 12 March 2015

\begin{abstract}
We described a novel handheld device (termed FMTPen) for three-dimensional (3D) fluorescence molecular tomography (FMT). The FMTpen is characterized by its bendable structure and miniaturized size (10 $\mathrm{mm}$ in diameter) that can be potentially used as an intraoperative tool for the detection of tumor margins and for image-guided surgery. Several phantom experiments based on indocyanine green (ICG), an FDA approved near-infrared (NIR) fluorescent dye, were conducted to evaluate the imaging ability of this device. Two tumor-bearing mice were systematically injected with tumor-targeted NIR fluorescent probes (NIR-830-ATF68-IONP and NIR-830-ZHER2:343-IONP, respectively) and were then imaged to further demonstrate the ability of this FMT probe for imaging small animals.
\end{abstract}

Keywords: fluorescence molecular tomography (FMT); intraoperative optical imaging; targeted optical nanoparticle imaging probes

\section{Introduction}

Surgery is one of the most effective and widely used procedures in treating different forms of human cancers, in which accurate identification of tumor margins play a critical role to the results of surgery treatment. One of the major challenges in the surgery is that often surgeon fail to remove the entire tumor, leaving behind tumor-positive margins, metastatic lymph nodes, and/or satellite tumor nodules. Ultrasound (US), magnetic resonance imaging (MRI) and computed tomography (CT) have 
been widely used clinically to detect these various cancers [1-4]. Although each of these traditional imaging techniques has its advantages, they usually have low specificity and sensitivity in the detection of small tumors, or poor temporal resolution to achieve really-time imaging in surgery.

Over the past several years, there has been exponential increase in the use of non-targeted or targeted exogenous fluorescence makers in clinical applications and biological research. It also has prompted the development of several fluorescence imaging approaches [5-10]. A common fluorescence imaging method is the planar fluorescence reflectance imaging (FRI), which was developed to in vivo or ex vivo image fluorescence in animal or human models of various diseases [11-13]. Several recent studies have shown the clinical importance of this technique for intraoperative tumor imaging and image-guided surgery [14-17]. Despite the simplicity of the operation, the applicability of this technique has been limited by the poor spatial and depth resolutions. To overcome these limitations, near-infrared fluorescence molecular tomography (FMT) has been developed and evolved as an important molecular imaging tool $[18,19]$. Being a convenient and cost-effective optical imaging modality, FMT can provide accurate visualization and quantification of the three-dimensional (3D) distribution of fluorescent targets deep inside turbid tissue. So far, FMT has been widely used in biomedical research and clinical studies including cancer imaging, drug delivery, stem cell trafficking, and enzyme activity monitoring [20-23].

In this study, we present a novel FMT system based on a miniaturized handheld probe called the FMTPen, for intraoperative tumor imaging and image-guided surgery. This probe is highly suitable for the detection of small tumor lesions in the surgical cavity based on the miniaturized size (10 $\mathrm{mm}$ in diameter) and bendable structure. Several phantom and animal experiments have been performed to validate the imaging ability of this instrument.

\section{Materials and Methods}

The schematic of the handheld FMT probe system is shown in Figure 1. The laser beam generated by a continuous-wave (CW) $780 \mathrm{~nm}$ diode laser (Openxt, HL7301MG, Japan) was used as the light source. The excitation light beam from the laser diode (LD) was focused by a lens with $10 \mathrm{~cm}$ focus length (Thorlabs, NJ, USA), and sequentially delivered to each optical fiber in the source fiber array through a high-speed motorized scanning mirrors system (Thorlabs, GVS212, NJ, USA). For each source position, the emission fluorescence light from the detection fiber array was then collected by a $1024 \times 1024$ pixels deep-cooled CCD camera (Princeton Instrument, Trenton, NJ, USA) coupled with an $830 \mathrm{~nm}$ band-pass filter with $32 \mathrm{~nm}$ bandwidth (Edmund Optics, NJ, USA).

The insert in Figure 1a shows a photograph of the FMTPen, which is miniaturized with $10 \mathrm{~mm}$ diameter and equipped with a metal jacket outside to protect the optical fiber bundles when bending. Figure $1 \mathrm{~b}$ shows a photograph of the end face of FMTPen. The corresponding pattern of source and detector positions is shown in Figure 1c. As Figures 1b, c show, sixty-one optical fiber bundles are fixed with epoxy in a uniform arrangement, in which twenty-nine (red) are used as optical source and thirty-two (green) as detectors. The diameter of each optical fiber bundle is $0.8 \mathrm{~mm}$. For each experiment, the exposure time of the CCD camera was different depending on the intensity of the fluorescence intensity from the target. A binning of $4 \times 4$ pixels was then used to improve the 
signal-to-noise ratio (SNR). A Labview program was used to control the entire data acquisition. The data acquisition time is about 2 min depending on the exposure time of the CCD camera.

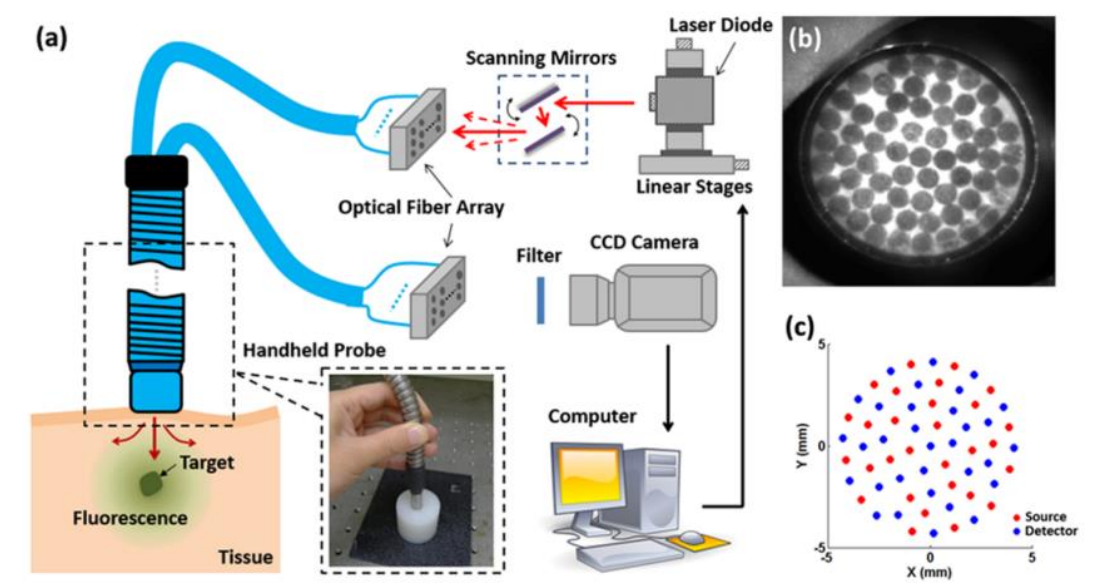

Figure 1. (a) Schematic of the FMTPen system; (b) photograph of the end face of FMTPen; and (c) corresponding arrangement of the sources (red) and detectors (blue).

Three-dimensional (3D) fluorescence images were reconstructed using an iterative finite element based algorithm that was described in detail previously [24-26]. Briefly, the finite element method is used to discretize the diffusion equations describing propagation of both excitation and emission light in tissue. This method also obtains the matrix representations capable of inverse problem solution. Fluorescence images are recovered by iteratively solving the forward equations and updating the optical/fluorescent property distributions from presumably uniform initial estimates of these properties. In this study, a 3D finite element mesh with 4928 nodes and 24660 tetrahedral elements was used for all the image reconstructions. The calculation required up to 8 minutes.

\section{Results and Discussion}

\subsection{Phantom Experiments}

To validate the ability of this FMTPen, several sets of phantom experiments using indocyanine green (ICG)-containing targets with different depth and contrast were performed. A cylindrical background phantom (30 cm in diameter and $20 \mathrm{~cm}$ in height), consisted of $\mathrm{TiO}_{2}$ solution, India ink, and $2 \%$ Agar powder, and had an absorption coefficient of $0.01 \mathrm{~mm}^{-1}$ and a reduced scattering coefficient of $1.0 \mathrm{~mm}^{-1}$. A small ICG-containing cylinder was placed at different depth of the background phantom to simulate the target.

Figure 2 shows the reconstructed FMT images (along transverse and sagittal planes) of a small cylindrical target ( $2 \mathrm{~mm}$ in diameter and $2 \mathrm{~mm}$ in height) with ICG concentrations of $2 \mu \mathrm{m}$ (a) and $5 \mu \mathrm{m}$ (b) imbedded at different depths (1 $\mathrm{mm}$ to $4 \mathrm{~mm}$ ). The black dashed circle or square in each image indicates the exact target location. From Figure 2, we can see that the target was well reconstructed at a depth of up to $4 \mathrm{~mm}$, while we note that the target size was overestimated slightly due to strong light scattering. The lateral resolution of FMTPen is $\sim 2 \mathrm{~mm}$. While this resolution is lower than some of the existing high resolution imaging modalities like CT and MRI, it is sufficient to 
enable surgeons to identify possible residual small tumor lesions in the cavity after surgery. The maximum depth resolution of this imaging probe $(\sim 5-6 \mathrm{~mm})$ is limited by the largest source-detector separation given in this probe. More discussion on spatial resolution of FMT can be found in our previous works [24,26,27].

(a) $2 \mu \mathrm{M}$
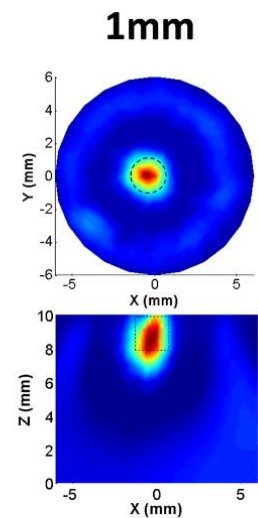

$\mathrm{x}(\mathrm{mm})$

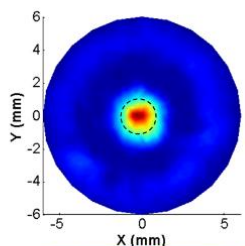

(b) $5 \mu \mathrm{M}$

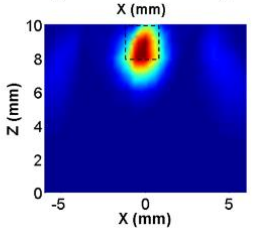

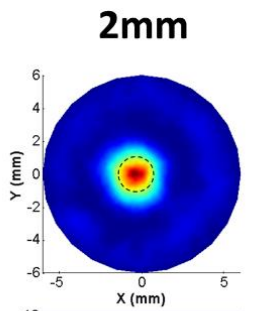
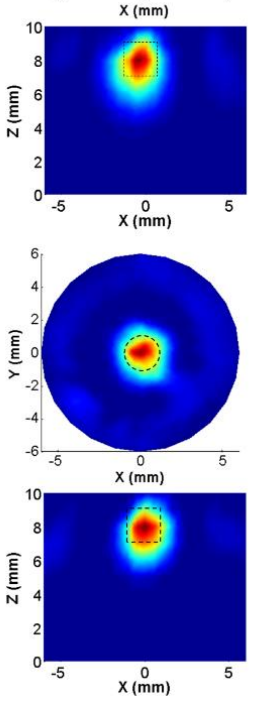
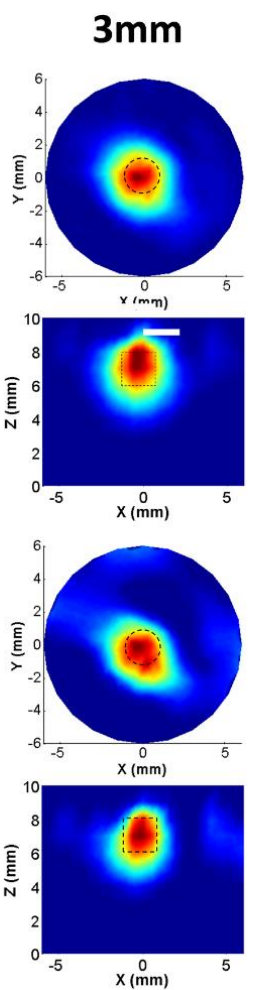

$4 \mathrm{~mm}$
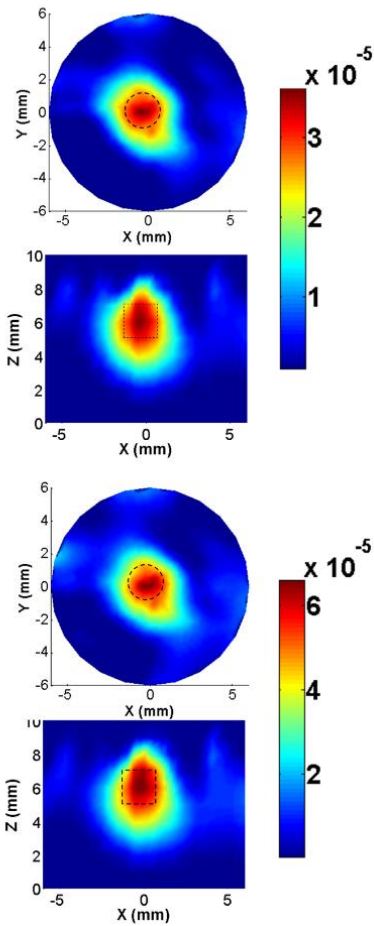

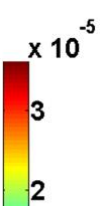

2

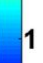

Figure 2. Fluorescence molecular tomography (FMT) images for a target having different indocyanine green (ICG) concentrations ((a) $2 \mu \mathrm{M}$ and (b) $5 \mu \mathrm{M})$ at different depths $(1 \mathrm{~mm}$ to $4 \mathrm{~mm}$ ).
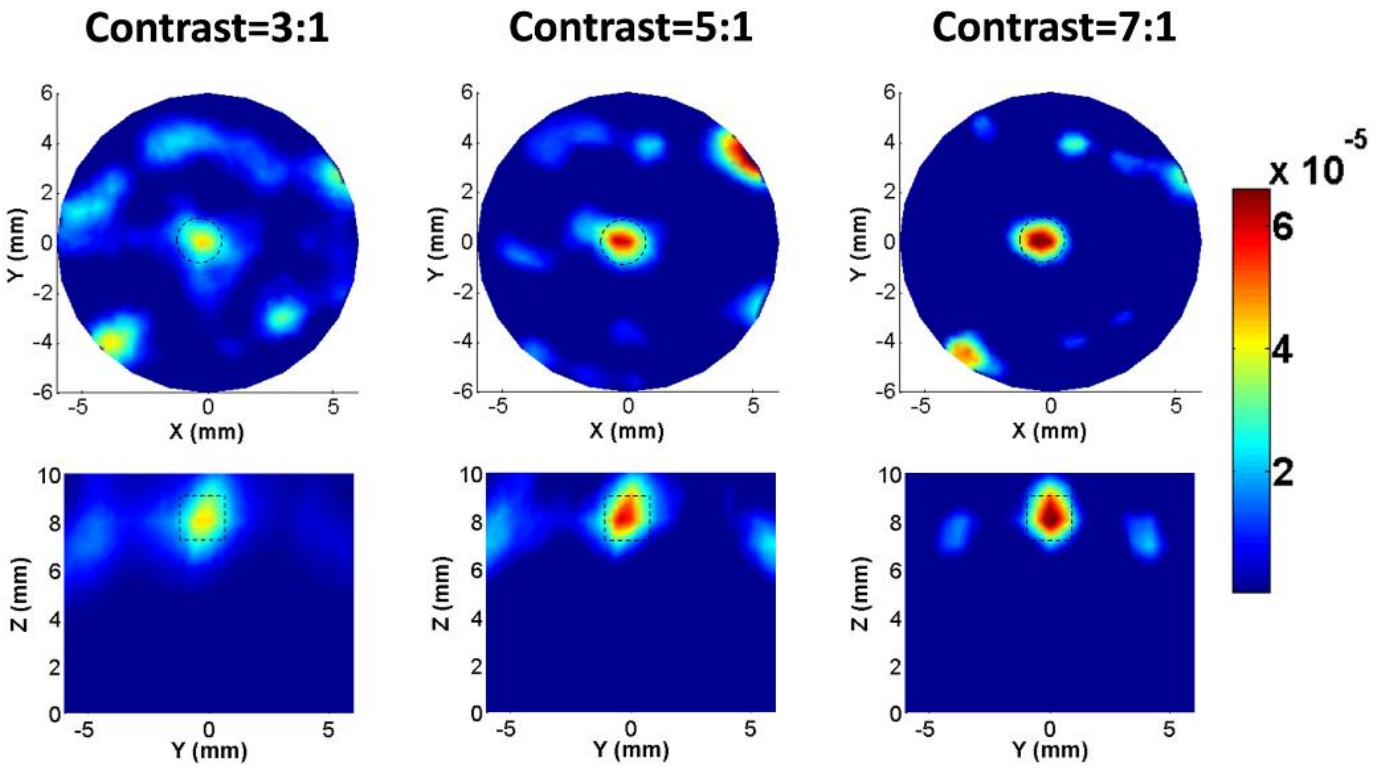

Figure 3. FMT images for a target having different ICG contrast $(3: 1,5: 1$ and $7: 1)$ relative to the background at a depth of $2 \mathrm{~mm}$. 
Figure 3 shows the reconstructed FMT images of a target having 3, 5 and 7 times ICG concentration relative to the background (target depth $=2 \mathrm{~mm}$ ). Againthe black dashed circle or square indicates the exact target location. As can be seen, the target can be well reconstructed even with an ICG contrast as low as 3:1. While some artifacts are observed in these cases, the imaging quality is improved with the increased ICG contrast.

\subsection{Animal Experiments}

To further demonstrate the imaging ability of this miniaturized FMT probe, two tumor-bearing mice with the use of different targeted contrast agents were imaged. An ovarian tumor model was established by injecting $5 \times 104$ of SKOV3 luciferase gene positive human ovarian cancer cells orthotopically (right side) and subcutaneously (left side), respectively.

Figure 4 shows the reconstructed FMT images along the transverse and sagittal planes for a tumor-bearing mouse Intraperitoneal (IP) injected with 290pmole NIR-830-ATF68-IONP, a peptide conjugated molecular probe loaded with both near-infrared dye and iron oxide nanoparticles that can target tumor cell receptors [28,29]. We can see that the tumors are well reconstructed in both cases. The outcomes also show that the subcutaneous tumor has more distribution of targeted nanoparticles than orthotopic tumor.

\section{NIR-830-AFT68-IONP}

Subcutaneous tumor
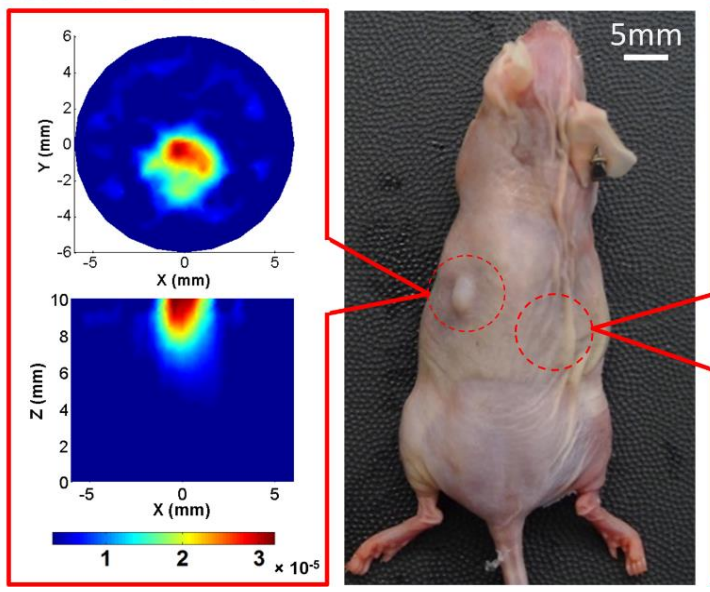

Orthotropic tumor

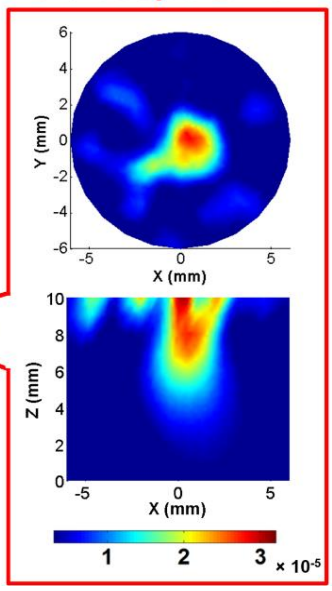

Figure 4. FMT images of a tumor-bearing mouse using NIR-830-ATF68-IONP: photograph of the mouse (middle), transverse and sagittal FMT slices of subcutaneous tumor (left), transverse and sagittal FMT slices of orthotropic tumor (right).

Figure 5 presents reconstructed FMT images along transverse and sagittal planes for a tumorbearing mouse IP-injected with 1000pmole NIR-830-ZHER2:343-IONP. Our previous work has shown that near-infrared dye labeled ZHER2:342 conjugated IONPs (NIR-830-ZHER2:342-IONP) was specifically targeted to primary and metastatic tumors in an orthotopic human ovarian cancer xenograft model. These tumors produced strong imaging signals for FMT, photoacoustic tomography (PAT) and magnetic resonance imaging (MRI) [21,30]. In this study, two tumors were also well detected using FMTPen. 


\section{NIR-830-ZHER ${ }_{2: 343}-$ IONP}

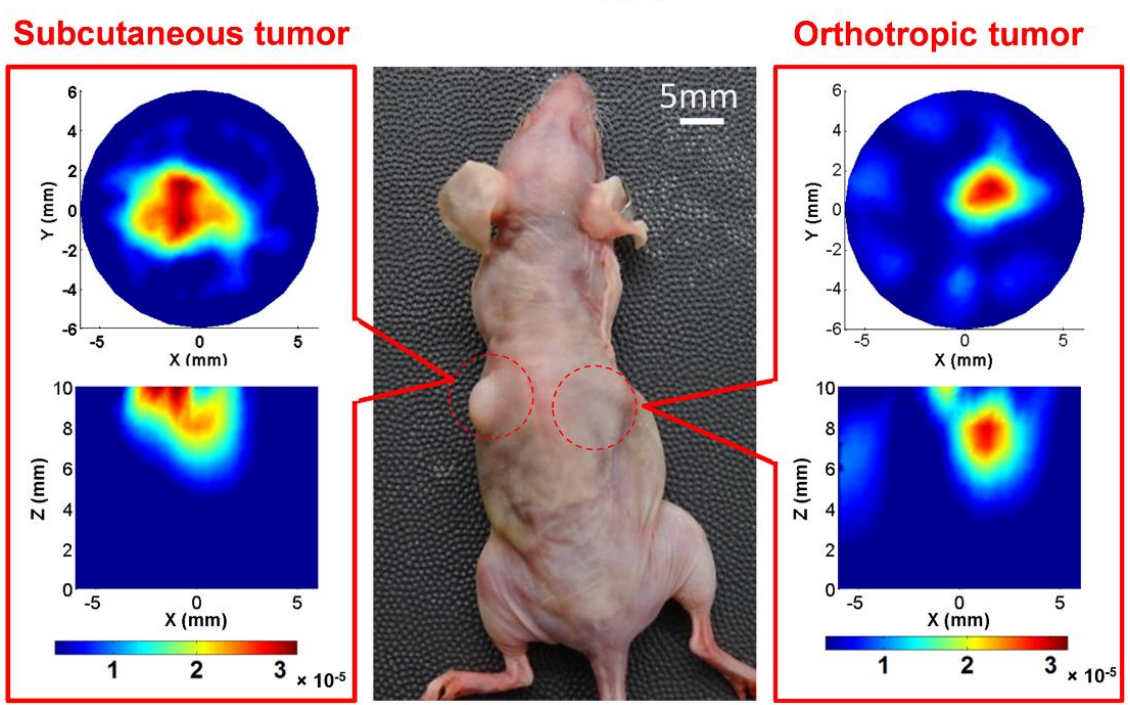

Figure 5. FMT images of a tumor-bearing mouse using NIR-830-ZHER2:343-IONP: photograph of the mouse (middle), transverse and sagittal FMT slices of subcutaneous tumor (left), (right) transverse and sagittal FMT slices of orthotropic tumor (right).

The primary advantage of FMTPen presented in this study is its miniaturized size (only $10 \mathrm{~mm}$ in diameter) and bendable structure, which makes this tool well suited for imaging in a small surgical cavity. Meanwhile, both the phantom and animal experiments have demonstrated that FMTPen has a good depth resolution of up to 5-6 mm and a high sensitivity of 3:1 contrast. Such depth resolution and sensitivity would enable a surgeon to find residual small tumor lesions that would be hard to be detected by the use of other imaging modalities. This is significant since the completeness of tumor removal during surgery is considered of utmost importance in achieving a cure. To the best of our knowledge, the FMTPen described here has been the smallest handheld FMT imaging probe so far. Due to these advantages, FMTPen offers a great potential to be an effective tool for intraoperative tumor imaging. By improving the rate of complete tumor removal in surgery, FMTPen would benefit a large number of patients. In addition, it would significantly reduce the cost in the management of cancer patients.

While the results shown are encouraging, we realize that there are still some improvements needed in order to translate this technique into human applications. The image reconstruction time was limited by CPU-based computer system. Graphic processing units (GPU) based parallel reconstruction algorithm can be developed to accelerate the image reconstruction, which was proved to be a powerful tool in our previous work [31]. In addition, the time desired for data acquisition needs to be significantly reduced to perform real-time image-guided surgery. Data acquisition time in this study, was limited by the exposure time of the CCD camera varying with target depth and contrast agent concentration to achieve optimal signal-to-noise ratio. In future, photomultiplier tubes (PMT) array can substitute for the CCD camera to speed up the data acquisition process due to their fast response time in microseconds and high sensitivity at low light levels. Despite these limitations, a more sophisticated and real-time intraoperative imaging technique is expected in the near future. 


\section{Conclusions}

In summary, we have presented a miniaturized FMT probe (FMTPen) and evaluated it with both phantom and animal experiments. The results obtained have shown that the FMTPen offers a great potential to develop a handheld probe for intraoperative tumor imaging due to its high sensitivity, miniaturized size $(1 \mathrm{~cm}$ diameter) and bendable structure. Our further goals are to apply the FMTPen for intraoperative imaging in breast cancer surgery.

\section{Acknowledgments}

This work was supported in part by the J. Crayton Pruitt Family endowment.

\section{Author Contributions}

Dr. Jiang is the corresponding author and directed this work. Hao Yang and Bin He designed the experiments; Hao Yang and Xianjin Dai performed the experiments; Minati Satpathy and Lily Yang prepared and provided the animals and nanoparticles; Hao Yang analyzed the data and wrote the paper, and all authors contributed to its improvement.

\section{Conflict of Interest}

The authors declare no conflict of interest.

\section{References}

1. Leroux, P.D.; Winter, T.C.; Berger, M.S.; Mack, L.A.; Wang, K.; Elliott, J.P. A comparison between preoperative magnetic resonance and intraoperative ultrasound tumor volumes and margins. J. Clin. Ultrasound 1994, 22, 29-36.

2. Trimble, E.L. The nih consensus conference on ovarian cancer: Screening, treatment, and follow-up. Gynecol. Oncol. 1994, 55, S1-S3.

3. Tammela, J.; Lele, S. New modalities in detection of recurrent ovarian cancer. Curr. Opin. Obstet. Gynecol. 2004, 16, 5-9.

4. Daisne, J.-F.; Sibomana, M.; Bol, A.; Cosnard, G.; Lonneux, M.; Grégoire, V. Evaluation of a multimodality image (ct, mri and pet) coregistration procedure on phantom and head and neck cancer patients: Accuracy, reproducibility and consistency. Radiot. Oncol. 2003, 69, 237-245.

5. Wagnieres, G.A.; Star, W.M.; Wilson, B.C. In vivo fluorescence spectroscopy and imaging for oncological applications. Photochem. Photobiol. 1998, 68, 603-632.

6. Richards-Kortum, R.; Sevick-Muraca, E. Quantitative optical spectroscopy for tissue diagnosis. Ann. Rev. Phys. Chem. 1996, 47, 555-606.

7. Valdés, P.A.; Leblond, F.; Kim, A.; Harris, B.T.; Wilson, B.C.; Fan, X.; Tosteson, T.D.; Hartov, A.; Ji, S.; Erkmen, K. Quantitative fluorescence in intracranial tumor: Implications for ala-induced ppix as an intraoperative biomarker. J. Neurosurg. 2011, 115, 11.

8. Gao, X.; Cui, Y.; Levenson, R.M.; Chung, L.W.; Nie, S. In vivo cancer targeting and imaging with semiconductor quantum dots. Nat. Biotechnol. 2004, 22, 969-976. 
9. Lee, J.-H.; Huh, Y.-M.; Jun, Y.-W.; Seo, J.-W.; Jang, J.-T.; Song, H.-T.; Kim, S.; Cho, E.-J.; Yoon, H.-G.; Suh, J.-S. Artificially engineered magnetic nanoparticles for ultra-sensitive molecular imaging. Nat. Med. 2007, 13, 95-99.

10. Yang, L.; Mao, H.; Cao, Z.; Wang, Y.A.; Peng, X.; Wang, X.; Sajja, H.K.; Wang, L.; Duan, H.; $\mathrm{Ni}, \mathrm{C}$. Molecular imaging of pancreatic cancer in an animal model using targeted multifunctional nanoparticles. Gastroenterology 2009, 136, 1514-1525.

11. Waldeck, J.; Häger, F.; Höltke, C.; Lanckohr, C.; von Wallbrunn, A.; Torsello, G.; Heindel, W.; Theilmeier, G.; Schäfers, M.; Bremer, C. Fluorescence reflectance imaging of macrophage-rich atherosclerotic plaques using an $\alpha v \beta 3$ integrin-targeted fluorochrome. J. Nucl. Med. 2008, 49, 1845-1851.

12. Josserand, V.; Texier-Nogues, I.; Huber, P.; Favrot, M.C.; Coll, J.-L. Non-invasive in vivo optical imaging of the lacz and luc gene expression in mice. Gene Therapy 2007, 14, 1587-1593.

13. Kircher, M.F.; Mahmood, U.; King, R.S.; Weissleder, R.; Josephson, L. A multimodal nanoparticle for preoperative magnetic resonance imaging and intraoperative optical brain tumor delineation. Can. Res. 2003, 63, 8122-8125.

14. Parungo, C.P.; Ohnishi, S.; Kim, S.-W.; Kim, S.; Laurence, R.G.; Soltesz, E.G.; Chen, F.Y.; Colson, Y.L.; Cohn, L.H.; Bawendi, M.G. Intraoperative identification of esophageal sentinel lymph nodes with near-infrared fluorescence imaging. J. Thorac. Cardiovasc. Surg. 2005, 129, 844-850.

15. Frangioni, J.V. In vivo near-infrared fluorescence imaging. Curr. Opin. Chem. Biol. 2003, 7, 626-634.

16. Nguyen, Q.T.; Olson, E.S.; Aguilera, T.A.; Jiang, T.; Scadeng, M.; Ellies, L.G.; Tsien, R.Y. Surgery with molecular fluorescence imaging using activatable cell-penetrating peptides decreases residual cancer and improves survival. Proc. Nat. Acad. Sci. 2010, 107, 4317-4322.

17. Themelis, G.; Yoo, J.S.; Soh, K.-S.; Schulz, R.; Ntziachristos, V. Real-time intraoperative fluorescence imaging system using light-absorption correction. J. Biomed. Opt. 2009, doi:10.1117/1.3259362.

18. He, B.; Xi, L.; Samuelson, S.R.; Xie, H.; Yang, L.; Jiang, H. Microelectromechanical systems scanning-mirror-based handheld probe for fluorescence molecular tomography. Appl. Opt. 2012, $51,4678-4683$.

19. Zhao, Q.; Jiang, H.; Cao, Z.; Yang, L.; Mao, H.; Lipowska, M. A handheld fluorescence molecular tomography system for intraoperative optical imaging of tumor margins. Med. Phys. 2011, 38, 5873-5878.

20. Bhaskar, S.; Tian, F.; Stoeger, T.; Kreyling, W.; de la Fuente, J.M.; Grazú, V.; Borm, P.; Estrada, G.; Ntziachristos, V.; Razansky, D. Multifunctional nanocarriers for diagnostics, drug delivery and targeted treatment across blood-brain barrier: Perspectives on tracking and neuroimaging. Part Fibre Toxicol 2010, 7, 3.

21. Xi, L.; Satpathy, M.; Zhao, Q.; Qian, W.; Yang, L.; Jiang, H. Her-2/neu targeted delivery of a nanoprobe enables dual photoacoustic and fluorescence tomography of ovarian cancer. Nanomed.: Nanotechnol. Biol. Med. 2014, 10, 669-677. 
22. Kossodo, S.; Pickarski, M.; Lin, S.-A.; Gleason, A.; Gaspar, R.; Buono, C.; Ho, G.; Blusztajn, A.; Cuneo, G.; Zhang, J. Dual in vivo quantification of integrin-targeted and protease-activated agents in cancer using fluorescence molecular tomography (fmt). Molecul. Imag. Biol. 2010, 12, 488-499.

23. Rudin, M.; Weissleder, R. Molecular imaging in drug discovery and development. Nat. Rev. Drug Dis. 2003, 2, 123-131.

24. Jiang, H. Frequency-domain fluorescent diffusion tomography: A finite-element-based algorithm and simulations. Appl. Opt. 1998, 37, 5337-5343.

25. Wu, C.; Barnhill, H.; Liang, X.; Wang, Q.; Jiang, H. A new probe using hybrid virus-dye nanoparticles for near-infrared fluorescence tomography. Opt. Commun. 2005, 255, 366-374.

26. Tan, Y.; Jiang, H. Diffuse optical tomography guided quantitative fluorescence molecular tomography. Appl. Opt. 2008, 47, 2011-2016.

27. Jiang, H. Diffuse Optical Tomography: Principles and Applications; CRC Press: Boca Raton, FL, USA, 2010.

28. Yang, L.; Peng, X.-H.; Wang, Y.A.; Wang, X.; Cao, Z.; Ni, C.; Karna, P.; Zhang, X.; Wood, W.C.; Gao, X. Receptor-targeted nanoparticles for in vivo imaging of breast cancer. Clin. Can. Res. 2009, 15, 4722-4732.

29. Yang, H.; Xi, L.; Samuelson, S.; Xie, H.; Yang, L.; Jiang, H. Handheld miniature probe integrating diffuse optical tomography with photoacoustic imaging through a mems scanning mirror. Biomed. Opt. Exp. 2013, 4, 427-432.

30. Satpathy, M.; Wang, L.; Zielinski, R.; Qian, W.; Lipowska, M.; Capala, J.; Lee, G.Y.; Xu, H.; Wang, Y.A.; Mao, H. Active targeting using her-2-affibody-conjugated nanoparticles enabled sensitive and specific imaging of orthotopic her-2 positive ovarian tumors. Small 2014, 10, 544-555.

31. Zhang, T.; Zhou, J.; Carney, P.R.; Jiang, H. Towards real-time detection of seizures in awake rats with gpu-accelerated diffuse optical tomography. J. Neurosci. Meth. 2015, 240, 28-36.

(C) 2015 by the authors; licensee MDPI, Basel, Switzerland. This article is an open access article distributed under the terms and conditions of the Creative Commons Attribution license (http://creativecommons.org/licenses/by/4.0/). 